\title{
Ultrassonografia abdominal pré-operatória em cães e gatos com suspeita de tumores abdominais
}

\author{
Preoperative Abdominal Ultrasonography in dogs and cats with suspected \\ abdominal tumors
}

\section{Daniela Aparecida Ayres Garcia ${ }^{{ }^{*}}$ Tilde Rodrigues Froes ${ }^{\mathbb{I}}$ Simone Domit Guérios ${ }^{\mathrm{I}}$}

\section{RESUMO}

\begin{abstract}
Objetivou-se com este estudo analisar a acurácia do exame ultrassonográfico na definição da origem de neoplasias abdominais e avaliar a eficiência da técnica em sugerir a malignidade do processo. Em 24 pacientes de 113 animais avaliados pelo exame ultrassonográfico, foi observado massa elou entremeado nodular em diferentes órgãos da cavidade abdominal, classificados em tipo I, II, II(+) e III. Compararam-se os achados ultrassonográficos com a cirurgia e o exame histopatológico. A determinação da origem da massa foi correta em 20 (83\%) animais. Pelos dados estatísticos de acurácia de 91,67\% obtidos, acredita-se que o exame ultrassonográfico é interessante para se determinar previamente a malignidade, principalmente naqueles pacientes que apresentam lesões agressivas.
\end{abstract}

Palavras-chave: ultrassom, neoplasia, malignidade, massa.

\section{ABSTRACT}

The aim of this study was evaluate efficacy of the ultrassonographic exam in the defining the location of the abdominal neoplasm and to estimate the accuracy of the method in screening the malignant neoplasm process. In 24 patients of 113 animals evaluated by the ultrassonographic exam it was observed a mass and/or a intermingled nodule in different organs in the abdominal cave, classified as type I, II, $I I(+)$ e III. It was compared the sonographic findings with surgical and histopathological analysis. The determination of the mass origin was correct in $20(83 \%)$ animals and the percentage accuracy of malignant ultrasound determination was $91.67 \%$. So, it is believed that the ultrasound is interesting to determine the malignancy in advanced cases, especially in those patients who have aggressive lesions.

Key words: ultrasound, cancer, malignant, mass.

\section{INTRODUÇÃO}

A prevalência dos tumores em cães e gatos tem aumentado nas últimas décadas. Essa condição pode resultar de um aumento real na incidência ou de um crescimento na população de pequenos animais com risco para o desenvolvimento das neoplasias. Além desses fatores, há a sensibilização e interesse dos proprietários de animais de estimação em prosseguir com diagnóstico e tratamento (PAOLONI \& KHANNA, 2007). O diagnóstico por imagem, importante auxiliar na oncologia veterinária, evoluiu substancialmente nos últimos anos, principalmente com a inclusão de novas metodologias, entre elas a ultrassonografia bidimensional e com doppler (BLEVINS, 2002). A ultrassonografia abdominal auxilia a estreitar o diagnóstico na definição da origem dos tumores e qual é o possível orgão envolvido, além de auxiliar no estadiamento, na monitorização e na avaliação das possíveis complicações dos tumores abdominais ou infiltrativos (BRAGG, 1985; FROES, 2004).

Os objetivos deste estudo foram analisar a acurácia do exame ultrassonográfico na definição da origem das neoplasias abdominais e avaliar a eficiência da técnica em apontar a malignidade do processo neoplásico.

'Departamento de Medicina Veterinária, Universidade Federal do Paraná (UFPR), 80035-050, Curitiba, PR, Brasil. E-mail: daniapag@yahoo.com.br.*Autor para correspondência. 


\section{MATERIAL E MÉTODOS}

Em período de 30 meses (Junho 2008 a Dezembro de 2010), foi realizado um estudo prospectivo, observacional e seccional descritivo no Hospital Veterinário da Universidade do Paraná e no Garra Hospital Veterinário. Foram analisados animais encaminhados ao serviço de ultrassonografia, seguindo os seguintes critérios de inclusão: cães e gatos de qualquer sexo e idade, com ou sem raça definida (CRD/SRD), nos quais, pelo exame ultrassonográfico, detectava-se massas e/ou entremeado nodular em diferentes órgãos da cavidade abdominal. Esses animais foram encaminhados para procedimento cirúrgico exploratório, para comparação entre os achados ultrassonográficos e os achados cirúrgicos, além da coleta das massas para análise histopatológica.

Para o experimento, foram utilizados dois aparelhos de ultrassonografia, sendo um Ultrasonix, modelo OP, e o outro aparelho da marca GE, modelo logiq expert 3 , com transdutores eletrônicos convexos e lineares, multifrequenciais, variando de 2,0 a 14,0 $\mathrm{MHz}$.

Os exames foram realizados por dois ultrassonografistas treinados. A técnica ultrassonográfica e a análise dos órgãos abdominais foram realizadas conforme os critérios indicados por MATTOON et al. (2004). Quando na presença de massas muito grandes, inicialmente avaliavam-se as suas características ultrassonográficas e, na sequência, ocorria a tentativa de localizar o órgão de origem, através de exclusão dos órgãos normais detectados durante o exame.

As lesões focais e multifocais em órgãos parenquimatosos e rins foram categorizadas durante $o$ exame como benigna, maligna e impossível de determinar de acordo com a característica de ecotextura apresentada, forma e grau de invasão apresentados, seguindo a classificação sugerida por FROES (2004). $\mathrm{Na}$ interpretação das imagens ultrassonográficas da vesícula urinária, foram determinadas a presença ou a ausência de alteração em parede do órgão. Essas adulterações foram classificadas como tipo II para aquelas que apresentavam lesões parietais infiltrativas e tipo III quando a alteração apresentava característica de massas vegetantes. As lesões do tipo II quando associadas à alteração em outro órgão (+) e as lesões do tipo III foram consideradas malignas, conforme demonstra a tabela 1 .

$\mathrm{Na}$ avaliação do trato gastrintestinal, foram verificadas: a espessura da parede, perda ou não da estratificação das camadas, o envolvimento de linfonodos mesentéricos e demais alterações na cavidade, como, por exemplo, o aumento da ecogenicidade do mesentério. Essas características foram determinadas e descritas. Lesões nas quais se notaram completa perda de estratificação parietal associada a espessamentos também foram classificadas como malignas, conforme sugerido por PENNINCK et al. (2003).

O resultado do exame ultrassonográfico foi apresentado a diferentes cirurgiões, que então executariam o procedimento cirúrgico para exérese das lesões e coleta das massas para análise histopatológica. Após a operação, os cirurgiões relatavam verbalmente se o exame ultrassonográfico auxiliou na conduta cirúrgica dos animais avaliados. Foram realizados testes de sensibilidade, especificidade, valor preditivo positivo e valor preditivo negativo pelo Diagnostic or Screening test evaluation, pelo Método Wilson Score, a fim de verificar a efetividade da classificação de malignidade pela técnica ultrassonográfica.

\section{RESULTADOS}

De 113 animais avaliados ultrassonograficamente, 24 (18 cães e 6 gatos) encontravam-se dentro do critério de inclusão do estudo, conforme demonstra a tabela 2. Com o método ultrassonográfico, o órgão abdominal (origem) acometido pela massa abdominal foi corretamente classificado em 20/24 casos, erroneamente classificados em 1/24 casos e não identificado em $3 / 24$ casos.

$\mathrm{O}$ caso de um gato com massa de origem prostática foi erroneamente classificado como vesical (caso 21). Em três casos, a origem não foi determinada pelo exame ultrasonográfico (casos 22, 23 e 24) devido à grande extensão das massas que dificultavam a correta visualização dos órgãos abdominais. No ultrassom, os tumores se apresentavam com tamanho maior que $15 \mathrm{~cm}$ de diâmetro, ecogenicidade heterogênea, com áreas anecóicas, hiperecóicas e "em alvo" entremeadas, todas foram classificadas como sendo do tipo III.

Pela análise histopatológica, em 19 animais, foi confirmada neoplasia maligna; em um caso, neoplasia benigna e; em quatro casos, as alterações não eram tumores, conforme tabelas 1 e 2 . Nos casos considerados benignos, somente o hematoma foi classificado como lesão tipo II (+), o que indicou malignidade pelo aspecto ultrassonográfico.

Nos animais que apresentavam alterações esplênicas, de acordo com a análise histopatológica, cinco casos eram hemangiossarcoma (caso 7, 8, 9, 10 e 11) e um era hematoma (caso 14). Ultrassonograficamente, o parênquima esplênico desses pacientes estava aumentado, com contorno 
Tabela 1 - Categorização utilizada para definição ou não de malignidade pela imagem ultrassonográfica dos órgãos parenquimatosos (baço, próstata e massas indefiníveis), rins e bexiga, e distribuição do número de casos considerando as avaliações ultrassonográfica e histopatológica.

\begin{tabular}{|c|c|c|c|c|c|}
\hline Categoria & Tipo I & Tipo II & Tipo II (+) & Tipo III & Lesões difusas \\
\hline Imagem correspondente & $\begin{array}{l}\text { Nódulos de } \\
\text { contorno } \\
\text { regular e } \\
\text { aspecto } \\
\text { homogêneo }\end{array}$ & $\begin{array}{l}\text { Nódulos ou } \\
\text { massas de } \\
\text { contorno } \\
\text { irregular, } \\
\text { hipoecóicoas, } \\
\text { hiperecóicas } \\
\text { ou em "alvo" }\end{array}$ & $\begin{array}{l}\text { Nódulos ou } \\
\text { massas de } \\
\text { contorno } \\
\text { irregular, } \\
\text { hipoecóicoas, } \\
\text { hiperecóicas } \\
\text { ou em "alvo" }\end{array}$ & $\begin{array}{l}\text { Alterações focais } \\
\text { ou multifocais de } \\
\text { ecogenicidade } \\
\text { mista - nódulos } \\
\text { em "alvo", } \\
\text { nódulos } \\
\text { cavitários com } \\
\text { calcificações, } \\
\text { que alteram o } \\
\text { contorno e/ou } \\
\text { cápsula do órgão }\end{array}$ & $\begin{array}{l}\text { Aumentos ou } \\
\text { reduções da } \\
\text { ecogeni-cidade }\end{array}$ \\
\hline Grau de complexidade & $\begin{array}{l}\text { Baixa } \\
\text { complexidade }\end{array}$ & $\begin{array}{l}\text { Média } \\
\text { complexidade }\end{array}$ & $\begin{array}{l}\text { Média } \\
\text { complexidade }\end{array}$ & $\begin{array}{l}\text { Alta } \\
\text { complexidade }\end{array}$ & - \\
\hline Definições & $\begin{array}{l}\text { Provável } \\
\text { benigno }\end{array}$ & $\begin{array}{l}\text { Suspeito para } \\
\text { maligno }\end{array}$ & $\begin{array}{l}\text { Provável } \\
\text { maligno }\end{array}$ & Maligno & $\begin{array}{l}\text { Impossível } \\
\text { determinar }\end{array}$ \\
\hline Avaliação ultrassom & Tipo I & Tipo II & Tipo II (+) & Tipo III & Lesões difusas \\
\hline Baço & 3 casos & - & 4 casos & 2 casos & - \\
\hline Próstata & - & - & 1 caso & - & - \\
\hline Massas indefinidas & - & - & - & 3 casos & - \\
\hline Rim & - & - & - & 3 casos & - \\
\hline Bexiga & - & - & 1 caso & 2 casos & - \\
\hline Avaliação histopatológica & Baço & Próstata & $\begin{array}{l}\text { Massas } \\
\text { indefinidas }\end{array}$ & Rim & Bexiga \\
\hline Benigno & 4 casos & - & 1 caso & - & - \\
\hline Maligno & 5 casos & 1 caso & 2 casos & 3 casos & 3 casos \\
\hline
\end{tabular}

(+) Indica lesão associada a: efusão peritoneal e/ou linfonodomegalia e/ou lesão em outro órgão. Os outros cinco casos restantes do estudo são os de neoplasia maligna em estômago e alças intestinais.

abaulado, irregular e com presença de uma massa de ecogenicidade mista. Em três animais (casos 8, 9 e 14), ainda se identificou moderada quantidade de líquido livre na cavidade abdominal, indicando sinais de ruptura da massa.

Nos casos 12 (nódulos de regeneração) e 13 (esplenite), observou-se que o parênquima esplênico estava discretamente aumentado de tamanho, contornos preservados e ecogenicidade difusamente heterogênea, com áreas anecóicas circulares entremeadas. No caso 15 (hiperplasia focal com áreas de necrose), ao exame ultrassongráfico, o baço estava aumentado de tamanho com contornos arredondados e apresentava aumento de ecogenicidade com presença de massa heterogênea, medindo $3,9 \times 2,9 \mathrm{~cm}$ e de contornos irregulares. No entanto, a presença de líquido livre abdominal não foi detectada.

Os achados ultrassonográficos e a classificação pré-determinada dos pacientes com carcinoma de células transicionais da bexiga foram: no caso 1 , verificou-se presença de massa infiltrativa e expansiva na parede com invasão para o colo vesical; no caso 2, observou-se na bexiga a presença de massa vegetante e irregular progredindo para o lúmen. Esses dois primeiros casos foram classificados como tipo III. Já o caso 3 foi classificado como tipo II (+), pois a bexiga demonstrava severo espessamento da parede vesical com perda da definição das camadas, associado à hidronefrose obstrutiva bilateral.

Observaram-se três casos de massas renais (casos 4, 5 e 6), determinados histopatologicamente como adenocarcinoma. Ao exame ultrassonográfico, os rins afetados estavam aumentados de tamanho com perda da arquitetura interna renal (Figura 1) e foram classificados como do tipo III, malignos.

Os achados ultrassonográficos nos casos de neoplasia gastrintestinal (casos 16, 17, 18, 19 e 20) foram espessamento parietal e perda da estratificação 
Tabela 2 - Descrição da característica clínica e epidemiológica dos cães e gatos, do órgão alvo acometido pela lesão previamente detectada pela ultrassonografia, sua classificação pelo exame ultrassonográfico e o seu tipo histopatológico.

\begin{tabular}{|c|c|c|c|c|c|c|}
\hline & & & & \multicolumn{3}{|c|}{ Sistema Urinário } \\
\hline № & Raça & Sexo & Idade & Órgão acometido & Tipo pelo ultrassom & Tipo histológico \\
\hline 01 & Poodle & Fêmea & 10 anos & Bexiga & Tipo III & $\begin{array}{l}\text { Carcinoma de células } \\
\text { transicionais }\end{array}$ \\
\hline 02 & P. Shetland & Fêmea & 9 anos & Bexiga & Tipo III & $\begin{array}{l}\text { Carcinoma de células } \\
\text { transicionais }\end{array}$ \\
\hline 03 & $\begin{array}{l}\text { Bloodhoun } \\
\text { d }\end{array}$ & Macho & 9 anos & Bexiga & Tipo II (+) & $\begin{array}{l}\text { Carcinoma de células } \\
\text { transicionais }\end{array}$ \\
\hline 04 & Fila & Fêmea & 9 anos & Rim direito & Tipo III & Adenocarcinoma renal \\
\hline 05 & Rottweiler & Fêmea & 7 anos & Rim esquerdo & Tipo III & Adenocarcinoma indiferenciado \\
\hline 06 & SRD Felino & Fêmea & 7 anos & $\begin{array}{l}\text { Rim esquerdo } \\
\text { Sistema }\end{array}$ & $\begin{array}{l}\text { Tipo III } \\
\text { Linfático }\end{array}$ & Adenocarcinoma renal \\
\hline 07 & $\begin{array}{l}\text { SRD } \\
\text { Canino }\end{array}$ & Macho & 7 anos & Baço & Tipo II (+) & Hemangiossarcoma \\
\hline 08 & P. Alemão & Macho & 7 anos & Baço & Tipo III & Hemangiossarcoma \\
\hline 09 & $\begin{array}{l}\text { SRD } \\
\text { Canino }\end{array}$ & Fêmea & 13 anos & Baço & Tipo III & Hemangiossarcoma \\
\hline 10 & $\begin{array}{l}\text { SRD } \\
\text { Canino }\end{array}$ & Fêmea & 9 anos & Baço & Tipo II (+) & Hemangiossarcoma \\
\hline 11 & Rottweiler & Macho & 12 anos & Baço & Tipo II (+) & Hemangiossarcoma \\
\hline 12 & SRD Felino & Macho & 11 anos & Baço & Tipo I & Nódulos regeneração \\
\hline 13 & Persa & Macho & 7 anos & Baço & Tipo I & Esplenite \\
\hline 14 & Teckel & Fêmea & 12 anos & Baço & Tipo II (+) & Hematoma \\
\hline \multirow[t]{2}{*}{15} & Poodle & Fêmea & 5 anos & Baço & Tipo I & $\begin{array}{l}\text { Hiperplasia focal com áreas de } \\
\text { necrose }\end{array}$ \\
\hline & & & & \multicolumn{3}{|c|}{ Sistema Gastrintestinal } \\
\hline 16 & Schnauzer & Fêmea & 12 anos & Alças intestinais & - & Leiomiossarcoma \\
\hline 17 & Pug & Macho & 5 anos & Alças intestinais & - & Sarcoma indiferenciado \\
\hline 18 & SRD Felino & Fêmea & 3 anos & Alças intestinais & - & Linfoma \\
\hline 19 & SRD Felino & Macho & 12 anos & Alças intestinais & - & Linfoma \\
\hline 20 & $\begin{array}{l}\text { SRD } \\
\text { canino }\end{array}$ & Macho & 3 anos & Estômago & - & Linfoma \\
\hline & & & & Sistema & Genital & \\
\hline 21 & SRD Felino & Macho & 13 anos & Próstata & Tipo II (+) & Adenocarcinoma indiferenciado \\
\hline 22 & Golden & Fêmea & 10 anos & $\begin{array}{l}\text { Ovário } \\
\text { Tecidos }\end{array}$ & $\begin{array}{l}\text { Tipo III } \\
\text { Moles }\end{array}$ & Disgerminoma \\
\hline 23 & Cocker & Fêmea & 12 anos & Indefinido & Tipo III & Sarcoma de tecidos moles \\
\hline 24 & Bulldog & Macho & 9 anos & Indefinido & Tipo III & Sarcoma de tecidos moles \\
\hline
\end{tabular}

das camadas com leve distensão fluida luminal indicativa de processo obstrutivo. Nos três casos de linfoma (casos 18, 19 e 20), esta perda da estratificação parietal era difusa com linfonodos ao redor aumentados de tamanho.

Pela análise estatística, confirmou-se que o exame ultrassonográfico foi $100 \%$ preciso em determinar a malignidade nos casos com neoplasia maligna (Valor Preditivo Positivo), houve 90,48\% de sensibilidade, $100 \%$ de especificidade, $91,67 \%$ de acurácia e $60 \%$ de valor preditivo negativo para tal.

\section{DISCUSSÃO}

Comprovou-se que o exame ultrassonográfico é um bom método de diagnóstico de massas abdominais e que traz importante contribuição no que se refere à determinação da origem da lesão, bem como na pré-determinação de malignidade em diferentes órgãos. Entretanto, o exame ultrassonográfico não deve ser utilizado para categorização histopatológica dos tumores.

Os erros na classificação da procedência da neoplasia foram atribuídos ao tamanho das massas 

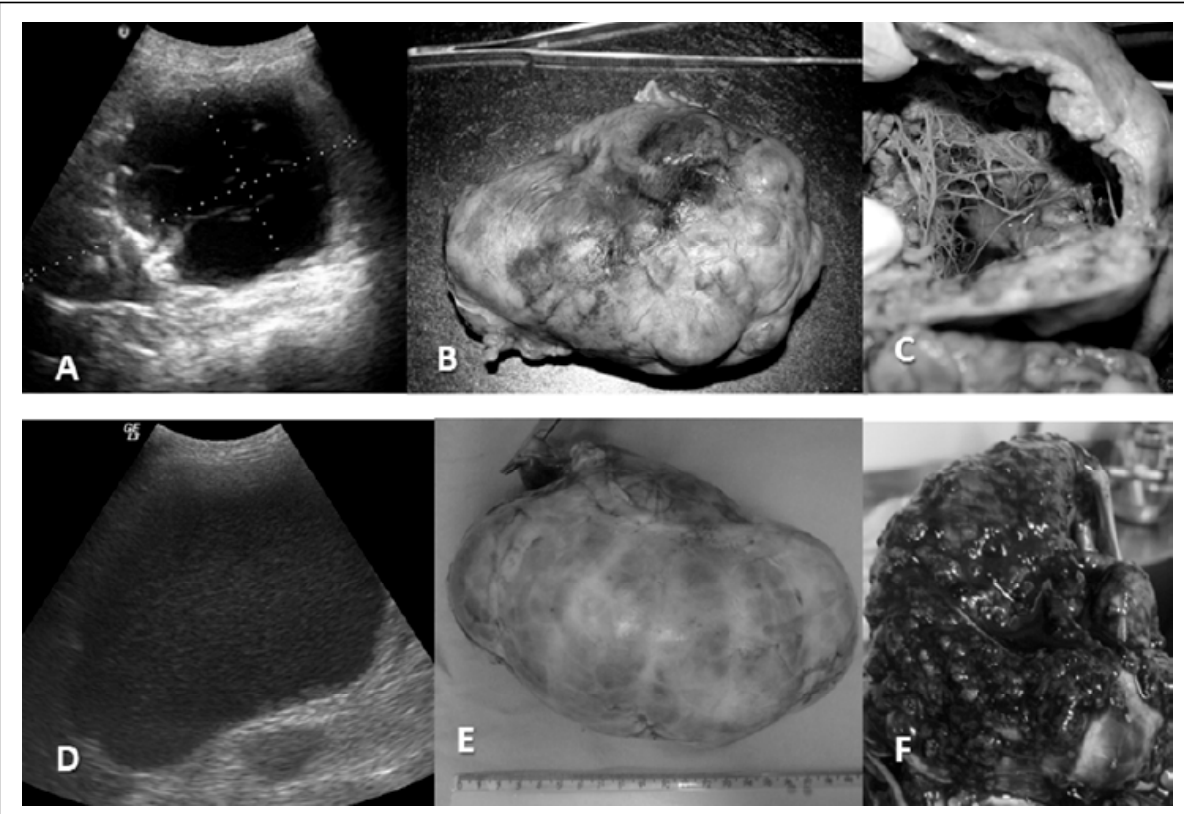

Figura 1 - (A) e (D) Imagens ultrassonográficas em plano sagital dos casos 4 e 5, nas quais observase perda da arquitetura interna renal, visualizada nos casos de adenocarcinoma. (B) e (E) Foto dos rins macroscopicamente e $(\mathrm{C})$ e $(\mathrm{F})$ da arquitetura interna macroscópica renal.

abdominais. Esses neoplasmas, quando extensos, podem tomar toda a cavidade abdominal, deslocando os demais órgãos de sua relação anatomotopográfica habitual, dificultando a determinação de sua origem (MILES, 1997). Tais dados foram comprovados nesta pesquisa, exemplos obtidos nos casos 22, 23, e 24, nos quais os tumores de grande extensão dificultaram a delimitação de sua origem. Outro fator que pode dificultar a determinação da origem de uma massa abdominal é quando ela é derivada de tecidos moles correlatos à cavidade peritoneal e não exatamente originada de um órgão, como, por exemplo, neoplasia do epiplon ou do peritônio. Assim como os sarcomas de tecidos moles que estão "livres" na cavidade, eventualmente aderidas a outros órgãos (CAMPBELL, 2009).

Vale ressaltar que a localização ultrassonográfica exata do órgão acometido por massas na cavidade abdominal é importante, pois auxilia o cirurgião no planejamento operatório, alertando-o sobre as dimensões do tumor, as prováveis aderências nos órgãos adjacentes e o leito de segmentos vasculares importantes que possam estar comprometidos. Esses dados foram mencionados pelos cirurgiões durante a execução dos procedimentos. Diante dessas constatações, pode-se dizer que o ultrassonografista tem papel fundamental em fornecer indícios dos locais da anatomia regional acometida, antecipando possíveis dificuldades táticas na cirurgia. Obviamente, quando o ultrassonografista não suprir essas questões, indicam-se outras modalidades imaginológicas, como a tomografia computadorizada e a ressonância magnética. Esses exames podem trazer mais informações, como a determinação de aderências e invasões em leitos vasculares, auxiliando melhor planejamento cirúrgico (SCHULTZ et al., 2009).

Acredita-se que haja associação direta da complexidade da lesão ultrassonográfica identificada e o aumento do risco de lesão maligna. Inclusive nas massas nas quais não foi possível determinar a sua origem, sugeriu-se uma pré-indicação de malignidade, auxiliando na propedêutica desses casos.

Com relação às alterações esplênicas, notase que a interferência quanto à malignidade deve ser feita com cautela, pois nem sempre o aspecto de alta complexidade é observado nos casos de hemangiossarcoma, conforme relatado por alguns pesquisadores (SMITH, 2003; IWASAKI et al., 2005). Apesar de o hemangiossarcoma esplênico apresentar comportamento maligno, em três de cinco pacientes, observou-se média complexidade e não foram achados correlatos que poderiam indicar a malignidade. Provavelmente, esses animais ainda estavam em fase inicial da doença, o que não permitiu a visibilidade dos achados ultrassonográficos já descritos na literatura. 
Dentre as neoplasias da bexiga, o carcinoma de células transicionais é o tipo histológico tumoral mais comum em cães (HENRY et al., 2003). As características das lesões tumorais já foram bem descritas na literatura (LEVEILLE, 1998) e o aspecto de massa vegetante é de fácil identificação, assim como foi visibilizado no cão 2 deste estudo. Entretanto, as lesões infiltrativas (animal 3) que provocam espessamentos difusos podem ser mais difíceis de serem diferenciadas de cistite crônica. Sempre que alterações como hidronefrose obstrutiva forem identificadas juntamente com os espessamentos difusos da bexiga urinária, deve-se suspeitar de malignidade. Uma vez que esse achado não é comum em pacientes que apresentam somente cistites crônicas, entende-se que esta anormalidade pode ser uma alerta ao ultrassonografista para indicação de técnicas mais invasivas de diagnóstico.

Nos casos de tumores renais, o exame ultrassonográfico foi essencial para a determinação do aumento no tamanho renal e da perda de definição do parênquima interno, sendo fundamental para o planejamento cirúrgico. Apesar de mencionada a determinação de malignidade para tais casos, acreditase que esse dado deve ser realizado com cuidado, já que as neoplasias renais podem ter características císticas e, ainda, abscedações em conjunto, dificultando tal objetivação (MOE \& LIUM, 1997).

Dos 113 animais avaliados ultrassonograficamente e cirurgicamente, cinco apresentaram tumor gastrintestinal, sendo o linfoma o mais observado. Em todos os casos, o exame ultrassonográfico contribuiu na localização e extensão das lesões, e os achados foram os mesmos encontrados por PENNINCK (1998) e FROES (2004). A ultrassonografia abdominal foi importantíssima nestes pacientes com suspeita de neoplasia gastrintestinal, permitindo eliminar diagnósticos diferenciais, principalmente com relação à doença inflamatória intestinal (MAGNE, 2000).

Houve erro na classificação da origem da massa prostática no caso 21. As prováveis causas incluem a difícil delimitação da massa devido à localização, que topograficamente ficava na região caudoventral da bexiga. Esse erro provavelmente surgiu devido à falta de conhecimento do aspecto ultrassonográfico dessa lesão, pois o número de casos de adenoarcinoma prostático em gatos descritos na literatura é pequeno (TURSI et al., 2008).

O cão número 22 , diagnosticado com digerminoma, tumor benigno no ovário esquerdo (JACKSON et al., 1985), apresentava massa de grande extensão, da qual não foi possível definir a origem. Ao exame ultrassonográfico, apresentava características de malignidade devido a sua característica sólida com nódulos entremeados e áreas com septações entremeadas. Apesar disso, mesmo que ao exame ultrassonográfico haja sinais de malignidade, deve-se sempre indicar a biópsia e a laparotomia exploratória para saber qual é o tipo histológico (MILES, 1997).

O exame ultrassonográfico é uma ferramenta eficaz ao sugerir malignidade, nota-se pelos resultados das análises de acurácia obtidos nessa pesquisa. A sensibilidade obtida foi de 90,48\% com Valor Preditivo Positivo de $100 \%$, ou seja, quando com características ultrassonográficas de agressividade e envolvimento de outros órgãos, a propensão para lesões malignas deve ser considerada positiva. Pondera-se que a maior dificuldade dessa determinação correlaciona-se a lesões consideradas pouco agressivas, talvez pelo tempo de instalação do processo, provavelmente o que interferiu no menor valor da sensibilidade, quando comparado à especificidade. Sendo assim, quando as lesões forem consideradas pouco agressivas ultrassonograficamente (tipo I e II), nunca se deve descartar a possibilidade de malignidade.

\section{CONCLUSÃO}

O exame ultrassonográfico é indicado no período pré-operatório de pacientes com massas abdominais, pois apresenta boa aplicabilidade na determinação da origem da lesão, auxiliando em apontar a malignidade dos processos. Contudo, nunca deve substituir as análises histopatológicas.

\section{CÔMITE DE ÉTICA}

Protocolo nํ016/2009.

\section{REFERÊNCIAS}

BLEVINS, W.E. Ultrasonography for cancer diagnosis and monitoring. In: MORRISON, W.B. Cancer in dogs and cats: medical and surgical management. 2.ed. Baltimore: Teton New Media, 2002. p.159-176.

BRAGG, D.G. Imaging strategies for oncologic diagnosis and staging. In: BRAGG, D.G. et al. Oncologic imaging. Oxford: Pergamon, 1985. p.13-21.

CAMPBELL, B.G. Primaryomental abscessation omental infarction in four dogs. Journal of the American Animal Hospital Association, v.45, p.168-175, 2009. Disponível em: < h t t p://www.jaa ha.org/cgi/reprint/4 5/4/ 168? maxtoshow $=\&$ hits $=10 \&$ RESULTFORMAT $=\&$ fulltext $=$ Primaryo mental+abscessation+omental+infarction+in+four+dogs\&search $\mathrm{id}=1 \&$ FIRSTINDEX $=0 \&$ resourcetype $=$ HWCIT $>$. Acesso em: 10 jan. 2011. 
FROES, T.R. Utilização da ultra-sonografia em cães com suspeitas de neoplasias do sistema digestório (fígado, intestinos e pâncreas). 2004. 155f. Tese (Doutorado em Cirurgia) - Faculdade de Medicina Veterinária e Zootecnia, Universidade de São Paulo, SP.

HENRY, J.C. et al. Evaluation of a bladder tumor antigen test as a screening test for transitional cell carcinoma of the lower urinary tract in dogs. American Journal of Veterinary Research, v.64, p.1017-1020, 2003. Disponível em: <http:/ / a v majournals a a ma.org/doi/abs/ 10.2460 / ajvr.2003.64.1017?prevSearch=allfield $\% 253 \mathrm{~A} \% 2528$ Evaluation $\% 2 \mathrm{Bo}$ f $\% 2$ B a $\% 2$ B b ladder $\% 2$ B t u m or $\% 2$ B a ntigen $\% 2$ Btest $\% 2$ B a s $\% 2$ B a $\% 2$ B s creening $\% 2$ Btest $\%$ 2Bfor\%2Btransitional $\% 2 \mathrm{~B}$ cell $\% 2 \mathrm{~B}$ carcinoma $\% 2 \mathrm{~B}$ of $\% 2 \mathrm{~B}$ the $\%$ 2Blower\%2Burinary\%2Btract\%2Bin\%2Bdogs\%2529\&searchHistoryKey=>. Acesso: 25 ago. 2010. doi: 10.2460/ajvr.2003.64.1017.

IWASAKI, M. et al. Aspectos ultra-sonográficos modo B e Doppler colorido nas alterações esplênicas focais e/ou multifocais de cães com suspeita de processos neoplásicos não linfóides. Clinica Veterinária, v.10, n.55, p.38-46, 2005.

JACKSON, M.L. et al. Ovarian dysgerminoma in a bitch. Canadian Veterinary Journal, v.26, p. 285-287, 1985. Disponível em: <http://www.ncbi.nlm.nih.gov/pmc /articles/ PMC1680110/pdf/canvetj00609-0061.pdf>. Acesso em: 28 dez. 2010.

LEVEILLE, R. Ultrasonography of urinay bladder disorders. Veterinary Clinics of North America: Small Animal Practice, v.28, n.4, p.799-821, 1998.

MAGNE, L.M. Gastrointestinal neoplasia. In: KIRK, R.W.; BONAGURA, J.D. Kirk current veterinary XIII small animal practice. 13.ed. Philadelphia: Saunders, 2000. p.675676.

MILES, K. Imaging abdominal masses. Veterinary Clinics of North America: Small Animal Practice, v.27, n.6, p.1403-1431, 1997.

MOE, L.; LIUM, B.J. Hereditary multifocal renal cystadenocarcinomas and nodular dermatofibrosis in 51 German shepherd dogs. Small Animal Practice, v.38, n 11, p.498-
505, 1997. Disponível em: <http://onlinelibrary.wiley.com/ doi/10.1111/j.17485827.1997. tb03306.x/abstract>. Acesso em: 21 nov. 2010. doi: 10.1111/j.1748-5827.1997.tb03306.x.

MATTOON, J.S. et al. Técnicas de varredura abodominal por ultra-som. In: NYLAND, T.G.; MATTOON, J.S. Small animal diagnostic ultrasound. 2.ed. Roca: São Paulo, 2004. Cap.5, p.53-84

PAOLONI, M.C.; KHANNA, C. Comparative oncology today. Veterinary Clinics of North America: Small Animal Practice, v.37, p.1023-1032, 2007. Disponível em: <http:// www.sciencedirect.com/science/article/pi i / S0195561607001118>. Acesso em: 12 ago. 2010. doi: 10.1016/ j.cvsm.2007.08.003.

PENNINCK, D.G. Characterization of gastrointestinal tumors. Veterinary Clinics of North America: Small Animal Practice, v.28, n.4, p.777-796, 1998.

PENNINCK, D.G. et al. Diagnostic value of ultrasonography in differentiating enteritis from intestinal neoplasia in dogs. Veterinary Radiology \& Ultrasound, v.44, n.5, p.570-575, 2003. Disponível em: <http://onlinelibrary.wiley.com/doi/ 10.1111/j.17408261.2003. tb00509.x/abstract>. Acesso em: 18 set. 2010 . doi: 10.1111/j.1740-8261.2003.tb00509.x.

SCHULTZ, R.M. et al. Contrast-enhanced computed tomography as a preoperative indicator of vascular invasion from adrenal masses in dogs. Veterinary Radiololgy \&Ultrasound, v.50, n.6, p.625-629, 2009.

SMITH, A.N. Hemangiosarcoma in dogs and cats. Veterinary Clinics of North America: Small Animal Practice, v.33, p. 533-552, 2003. Disponível em: <http://www.sciencedirect.com/ science/article/pii/S0195561603000020>. Acesso em: 14 ago. 2010. doi: 10.1016/S0195-5616(03)00002-0.

TURSI, M. et al. Adenocarcinoma of the disseminated prostate in a cat. Journal of Feline Medicine and Surgery, v.10, n.6, p.600-601, 2008. Disponível em: <http:// www.sciencedirect.com/science/article/pi i/ $\mathrm{S} 1098612 \mathrm{X} 0800082 \mathrm{X}>$. Acesso em: 10 out. 2010. doi: 10.1016/j.jfms.2008.02.008. 